\title{
Diferencias en el uso estratégico del espacio en categorías infantil y cadete de fútbol: una aplicación del análisis de coordenadas polares
}

\author{
Differences in the Strategic Use of Space in Under 14 and \\ Under 16 Soccer: A Polar Coordinate Analysis
}

\section{Diferenças na utilização estratégica do espaço nos escalóes de iniciados e juvenis no futebol: uma aplicação da análise de coordenadas polares}

Ibon Echeazarra Escudero ${ }^{1 *}$, Julen Castellano Paulis ${ }^{1}$, Oidui Usabiaga Arruabarrena ${ }^{1}$ y Antonio Hernández Mendo ${ }^{2}$

${ }^{1}$ Universidad del País Vasco (UPVIEHU), ${ }^{2}$ Universidad de Málaga

Resumen: Debido a la interacción inherente al fútbol, el uso del espacio social puede ser un aspecto clave en la dinámica del juego al que debería atenderse con especial atención. En esta línea, el presente estudio pretende comparar cómo es gestionado el espacio por equipos de diferente nivel competitivo. Para ello, doce partidos de equipos con jugadores infantiles y cadetes, seis por categoría, fueron observados, codificados y registrados a partir de un sistema taxonómico diseñado ad hoc (Sistema de Observación de la Acción de Juego en el Fútbol Base ó SOFBAS v.2). Superada la prueba de calidad del dato, a partir de los registro se implementó la técnica de coordenadas polares en su variante de retrospectividad genuina. Se escogieron como conductas focales o criterio las categorías correspondientes al espacio de juego efectivo (EJE), que representa la ubicación y contextualización de la acción del jugador con balón en relación al resto de jugadores participantes (compañeros y adversarios), para los que se distinguieron FF (Fuera-Fuera), FD (Fuera-Dentro), DF (Dentro-Fuera) y DD (Dentro-Dentro). Los resultados del estudio mostraron diferencias en el uso estratégico del espacio entre ambas categorías, presentando el juego de los cadetes un mayor equilibrio entre las acciones realizadas dentro y fuera del EJE. Con este trabajo los entrenadores podrían considerar adaptar las estrategias de entrenamiento más adecuadas a cada etapa evolutiva, con las que guiar de una manera más eficiente el proceso de formación de los participantes del fútbol formativo.

Palabras clave: Fútbol, formación, metodología observacional, coordenadas polares.

Abstract: Player interaction is an inherent part of soccer and the collective use of space may be an aspect of game dynamics that merits closer study. This study investigates differences in the use of playing space between under-14 and under-16 male soccer players. Twelve matches (six per age group) were observed, coded, and recorded using the ad hoc soccer observation instrument SOFBAS (version 2). Following confirmation of the quality of the data, the datasets were analyzed by polar coordinate analysis from the perspective of genuine retrospectivity. Effective playing area (EJE) categories were chosen as the criterion, or focal, behaviors. These categories represented the location of the player on his first contact with the ball in relation to the EJE and to the location of his teammates and opposing players. Four categories were used: FF (outside-outside), FD (outside-inside), DF (inside-outside), and DD (inside-inside). The polar coordinate analysis revealed differences in the strategic use of space between the two age groups, with the older players showing a more balanced use of the areas inside and outside the EJE. The results of this study suggest that training strategies adapted to the age and abilities of players might result in more efficient and effective training sessions and learning. Keywords: Soccer, training, observational methodology, polar coordinates. Resumo: Devido à interação inerente ao futebol, a utilizaçáo do espaço social pode ser um aspecto chave na dinâmica do jogo a que se deveria dar uma atençáo especial. Nesta linha, o presente estudo pretende comparar como é gerido o espaço por equipas de diferente nivel competitivo. Para isso, doze jogos de equipas de iniciados e juvenis, seis por cada escalão, foram observados, codificados e registados a partir de un sistema taxonómico construído ad hoc (Sistema de Observação da Açâo de Jogo no Futebol Base ou SOFBAS v.2). Ultrapassada a prova de qualidade dos dados, a partir dos registos implementou-se a técnica de coordenadas polares na sua variante de retrospetividade genuína. Escolheram-se como condutas focais ou critério as categorias correspondentes ao espaço de jogo efectivo (EJE), que representam a localização e o contexto da acção do jogador com bola em relaçáo aos outros jogadores envolvidos (companheiros e adversários), tendo-se distinguido FF (Fora-Fora), FD (Fora-Dentro), DF (DentroFora) y DD (Dentro-Dentro). Os resultados do estudo mostraram diferenças na utilização estratégica do espaço entre ambas as categorías, apresentando o jogo dos juvenis um maior equilíbrio entre as açóes realizadas dentro e fora do EJE. Com este trabalho os treinadores poderiam considerar adotar as estratégias de treino mais adequadas a cada etapa evolutiva, de modo a conduzir de modo mais eficaz o processo de formação dos praticantes do futebol jovem.

Palavras chave: Futebol, formação, metodología observacional, coordenadas polares.

\section{Introducción}

La búsqueda de nuevos y eficaces métodos de preparación en el fútbol es, desde hace tiempo, una de las principales

Dirección para correspondencia [Correspodence address]: Ibon Echeazarra Escudero. C/ Margarita Nelken 5, 1B. 01015. Vitoria-Gasteiz (Espańa). preocupaciones para entrenadores y técnicos que se dedican a la enseńanza de este deporte, experimentando una importante evolución en los últimos años (Bangsbo, Mohr y Krustrup, 2006; Reilly, 2009). En este sentido, la idea de que los programas de enseñanza del fútbol tienen que elaborarse a 
partir de las características del mismo deporte (Reilly, 2005), tiene cada vez más calado entre los entrenadores, que tratan de aplicar métodos de trabajo más acordes a los rasgos del juego. Sin embargo, son escasos los trabajos encaminados al estudio de estos aspectos en la vertiente más formativa de este deporte, el fútbol de base, siendo insuficiente el conocimiento en torno a este ámbito (Horn, 2002; Morris, 2000; Vegas, 2006). La posibilidad de construir y ampliar el conocimiento científico en el fútbol formativo pasa, en gran medida, por conocer las características del juego bajo las que operan los jugadores (Carling, Williams y Reilly, 2005), identificar los patrones de comportamientos que pueden ser esperados por los jugadores de una etapa formativa concreta e incluso encontrar sus posibilidades de evolución (Wein, 2007). Resulta por tanto esencial, profundizar en las demandas del juego, especialmente en las de tipo estratégico-conductual (Carling, 2013), a fin de configurar posibles estrategias de intervención que favorezcan el progresivo desarrollo de la competencia futbolística.

En relación a la observación de la acción de juego en fútbol resulta especialmente interesante abordar su estudio contemplando la complejidad del juego. Profundizar, por tanto, en el análisis de aspectos relativos a la interacción entre jugadores, que permitan identificar las dinámicas y patrones de organización característicos de los diferentes niveles de juego (Folgado, Lemmink, Frencken y Sampaio, 2012; Sampaio y Maças, 2012). El análisis de la complejidad, desde el estudio de la diada formada por los dos equipos en interacción, ha sido abordada por diferentes autores (Araujo, Davids, y Hristovski, 2006; Davids, Araújo, y Shuttleworth, 2005; Duarte et al., 2010; Perl, 2006; Suzuki y Nishijima, 2004) a partir del estudio de las distancias entre participantes, entre el balón y el objetivo de marca, la diferencia en cuanto al número de jugadores, velocidad del balón, etc., todos ellos buscando describir los comportamientos colectivos de los equipos. En el fútbol, las oportunidades de acción surgen de la complementariedad de las relaciones de los jugadores sobre el terreno de juego y, todo ello, en un entramado dinámico. Desde que Gréhaigne (1992) y Gréhaigne, Bouthier y David (1997) propusieran hace dos décadas el concepto de espacio de juego efectivo (EJE), esta perspectiva para comprender la relación de fuerzas sigue vigente (Gréhaigne, Godbout y Zerai, 2011). En todo este período, varios trabajos han propuesto operativizar esta relación de fuerzas desde una perspectiva sistematizada y a partir de la construcción de andamiajes conductuales en el marco de la metodología observacional. Desde las aportaciones de Castellano (2000) con los contextos de interacción, y Seabra y Dantas (2006) incorporando novedades respecto a la ubicación de los jugadores dentro o fuera del EJE, varios son los autores que lo han aplicado al análisis de diferentes competiciones (Barreira, Garganta, Castellano, Prudente y Anguera, 2014; Camerino, Chaverri, Anguera y
Jonsson, 2011; Castellano, Hernández-Mendo, Morales-Sánchez y Anguera, 2007; Castellano, Perea y Blanco-Villaseñor, 2007; Castellano, Perea y Hernández-Mendo, 2007, 2008; Perea, Castellano, Alday y Hernández-Mendo, 2012; Robles, Castellano, Perea, Martínez-Santos y Casamichana, 2011), aportando todos ellos relevantes aspectos del juego que ponen de manifiesto cómo se desarrollan las dinámicas sociales en el terreno de juego.

Algunos trabajos previos ya han utilizado la técnica de coordenadas polares (Sackett, 1980) para la descripción de las relaciones que fluyen en los deportes colectivo (Castellano, Hernández-Mendo y Haro, 2002; Hernández-Mendo y Anguera, 1999; Robles y Castellano, 2012 y 2014). También, de manera particular, esta técnica analítica ha sido utilizada para el análisis de la contextualización de las conductas desplegadas por los jugadores con balón a partir del criterio EJE (Castellano, 2000), con resultados satisfactorios en su aplicación a la comprensión de las dinámicas del juego (Castellano y Hernández-Mendo, 2002, 2003; Perea, 2008; Perea, 2008; Perea, Alday y Castellano, 2007; Robles, 2012). Esta particular técnica analítica, propia de la Metodología Observacional, posibilita conocer el tipo de relaciones que se producen entre las conductas correspondientes al sistema taxonómico propuesto, actuando como un potente reductor de datos. Este análisis posibilita construir mapas de relaciones entre diferentes categorías de conducta (vínculo entre la categoría focal y las condicionadas) donde se complementa una perspectiva diacrónica o en el transcurso del tiempo hacia delante y una retrospectiva o hacia atrás, consiguiendo un sistema de vectores por el cuál se puede mantener sin distorsión una elevada capacidad informativa.

Por todo lo expuesto hasta ahora, en el presente estudio se pretende llevar a cabo un análisis del espacio de juego efectivo a partir de la técnica de coordenadas polares, a fin de establecer rasgos que caractericen los aspectos del juego en el fútbol formativo (categorías de infantil y cadete). El diferente peso y distribución del juego por dentro y fuera del EJE, podría suponer un rasgo diferenciador de los diferentes niveles de competencia futbolística, ayudando a valorar la progresión del joven futbolista en su camino por adquirir la competencia específica de este deporte.

\section{Método}

\section{Diseño}

El estudio se enmarca en un diseño observacional perteneciente al cuadrante III (Anguera, Blanco-Villaseñor, Hernández-Mendo y Losada, 2011). Esto supone que se trata de un estudio de carácter nomotético (se observan varios jugadores de varios equipos), multidimensional (debido a que se combina un sistema mixto de observación a partir de formatos de 
campo y sistemas de categorías, abarcando diferentes dimensiones del juego) y puntual (motivado por que se observan varios partidos de una misma temporada sin la pretensión de hacer un seguimiento intersesional sobre la evolución de los jugadores o equipos), habiendo seguimiento intrasesional).

\section{Participantes}

En el estudio tomaron parte deportistas en proceso de formación, correspondientes a las categorías infantil (12-14 años) y cadete (14-16 ańos), que competían en la modalidad de fútbol 11. La unidad de observación empleada en la codificación fue la posesión individual del jugador con balón, la cual fue caracterizada por una herramienta multidimensional que más tarde se aborda. La codificación del conjunto de partidos grabados supuso el registro de aproximadamente 12.000 secuencias de multieventos (Bakeman y Quera, 2011) y, en consecuencia, más de 120.000 eventos. Por otro lado, el estudio cuenta con la aprobación institucional por parte del Comité de Ética de la Universidad del País Vasco (CEISH) para la realización del estudio.

\section{Instrumentos}

Instrumento de observación

Para describir las acciones técnico-tácticas desplegadas por los jugadores se hizo uso de la herramienta de observación SOFBAS v. 2 (Echeazarra, 2014). Se trata de una herramienta expresamente diseñada para la observación del fútbol de formación, a través de la combinación de sistemas de categorías y formatos de campo (Anguera, 1992), siguiendo la línea de otros trabajos que también han estudiado la acción de juego en fútbol (Ardá, 1998; Castellano, 2000; Hernández-Mendo, 1996; Perea, 2008). A partir de los 9 criterios y 73 categorías que la configuran, es posible identificar analizar aspectos tales como la conducta desplegada por el jugador que interviene con el móvil, la superficie de contacto con la que desarrolla la acción, el lugar de inicio y fin de la misma, la dirección que imprime al balón, así como su ubicación en relación al resto de jugadores (para más información se puede consultar Echeazarra, 2014). En el presente trabajo solo se contempla el estudio del criterio Espacio de Juego Efectivo, considerado un elemento de especial relevancia por su carácter innovador y por contemplar la interacción entre jugadores. En la siguiente tabla se describen y definen las cuatro categorías empleadas en el presente estudio: FF (Fuera-Fuera), FD (Fuera-Dentro), DF (Dentro-Fuera) y DD (Dentro-Dentro).
Tabla 1. Descripción de las categorías planteadas para el criterio Espacio de juego efectivo.

FF - Fuera-fuera: el jugador se encuentra en el perímetro formado por los jugadores de su equipo más alejados del centro, y fuera del perímetro del equipo contrario, cuando contacta por primera vez con el balón

FD - Fuera-dentro: el jugador se encuentra en el perímetro formado por los jugadores de su equipo más alejados del centro y dentro del perímetro del equipo contrario, cuando contacta por primera vez con el balón

DD - Dentro-dentro: el jugador se encuentra dentro del perímetro formado por los jugadores de su equipo más alejados del centro, y del perímetro del equipo contrario, cuando contacta por primera vez con el balón

DF - Dentro-fuera: el jugador se encuentra dentro del perímetro formado por los jugadores de su equipo más alejados del centro, y fuera del perímetro del equipo contrario, cuando contacta por primera vez con el balón

Instrumento de registro

Para la grabación de los partidos, se utilizó una cámara de vídeo modelo JVC GZ-MG730, apoyada sobre un trípode y situada en una de las esquinas de la balconada del campo. Para la codificación y registro de las conductas de los jugadores, se hizo uso del programa informático MOTS (Castellano, Perea, Alday y Hernández-Mendo, 2008), al que se incorporó la herramienta de observación SOFBAS v.2. Para el correcto visionado de los vídeos en el MOTS fue necesaria la conversión del dispositivo digital en formato ${ }^{*}$.mod (en el que graba la cámara) al formato *.mpeg, y se hizo a través de la aplicación informática Any Video Converter.

\section{Instrumento de análisis}

Con el objetivo de analizar la calidad del dato se empleó tanto el software Excel como el SDIS-GSEQ v5.1. (Bakeman y Quera, 1996, 2011). Para aplicar los análisis de generalizabilidad se implementó en las aplicaciones SAS v.9.1 (SAS Institute Inc., 1999) y SAGT v1.0 build 218.1 (Hernández-Mendo, Ramos-Pérez y Pastrana, 2012). Para implementar el análisis de coordenadas polares con los que configurar los mapas socioconductuales del uso estratégico del espacio se exportaron los datos registrados al programa informático HOISAN v.1.6 (Hernández-Mendo, López-López, Castellano, Morales y Pastrana, 2012).

\section{Procedimiento}

A partir de un protocolo de observación los observadores fueron formados para la codificación y registro de los partidos. Al final del proceso se calculó la calidad del dato, siendo los 
resultados favorables en cuanto a la concordancia intra e inter observadores (Echeazarra, Castellano y Usabiaga, 2013). Posteriormente, se grabaron un total de 20 partidos de competición oficial, 10 por categoría, a partir de los que, de manera aleatoria, se inició la codificación uno a uno. Se realizó un estudio apriorístico G (a partir de la Teoría de la Generalizabilidad) para conocer la muestra necesaria a partir de estimar la precisión en la generalización de dos partidos codificados. El binomio coste/beneficio guió la decisión del investigador para optar por un coeficiente de generalizabilidad óptimo (lo más próximo a 1). Además, en la línea de estudios previos (Blanco-Villaseñor, Castellano y Hernández-Mendo, 2000; Blanco-Villaseñor, Castellano, Hernández-Mendo, SánchezLópez y Usabiaga, 2014; Casamichana, Castellano, BlancoVillaseñor y Usabiaga, 2012; Usabiaga, Castellano, BlancoVillaseñor y Casamichana, 2013) en el ámbito observacional, se comprobó si el modelo cumplía los requisitos de normalidad, linealidad y homocedasticidad. Se aplicaron de manera específica los procedimientos VARCOMP Tipel y MIXED REML, con los que se pudo comprobar, efectivamente, que la varianza error en ambos procedimientos fue la misma, por lo que se cumplían los requisitos anteriormente mencionados.

Una vez que se obtuvieron los registros en formato Excel se exportaron, junto con la herramienta de observación, a la aplicación HOISAN v1.6 con las que se llevó a cabo los análisis secuenciales y, posteriormente, de coordenadas polares.

\section{Análisis de datos}

Para el análisis de datos se implementó la técnica de coordenadas polares, a partir de la cual se estimó un mapa de relaciones para cada una de las cuatro categorías del criterio espacio de juego efectivo y su relación entre ellas, complementando al mismo tiempo un punto de vista prospectivo y retrospectivo. Los aspectos clave para construir estos mapas son calcular el sumatorio de los residuos ajustados de los retardos de +1 a +5 en el plano prospectivo, el sumatorio de los residuos ajustados de los retardos de -1 a -5 en el plano retrospectivo, los ángulos, ratios y radios, con los que ubicarlos en uno de los cuadrantes: en el cuadrante I $(+,+)$ significa que la conducta criterio tiene una relación respecto a la condicionada de activación tanto en el plano prospectivo (o hacia adelante) como en el retrospectivo, b) el cuadrante II (+,-) significa que la conducta criterio excita en el plano retrospectivo pero no en el prospectivo, c) cuadrante III (-,-) significa que la relación entre la conducta criterio y la condicionada es de inhibición tanto hacia delante como hacia atrás, y d) cuadrante IV $(-,+)$ significa que la conducta criterio tiene una relación respecto a la condicionada de activación hacia delante e inhibición hacia atrás. Finalmente, el radio representa la intensidad de la relación entre pares de categorías (focal y condicional), y en donde un mayor valor significa mayor intensidad, siendo significativa por encima del valor crítico 1.96, o lo que es lo mismo, para $p<0.05$ (Anguera, 1997; Castellano y Hernández-Mendo, 2003; Hernández-Mendo y Anguera, 1998; Hernández-Mendo y Anguera, 1999; Perea, Castellano, Alday y Hernández-Mendo, 2012).

\section{Resultados}

Como puede apreciarse en las figuras de la 1 a la 4, la dinámica del juego respecto al uso del espacio de juego efectivo fue diferente en la comparación entre infantiles y cadetes, tanto en las relaciones que se establecieron entre las categorías (mismas categorías ubicadas en diferentes cuadrantes) como en la intensidad (radios de diferente magnitud) de sus relaciones.

En la figura 1 se muestra los mapas conductuales estimados a partir de la categoría focal DD (Dentro-Dentro). Como puede apreciarse, las relaciones fueron diferentes entre infantiles y cadetes, al estar ubicadas las categorías en cuadrantes distintos, a excepción de las categorías DD, donde para ambos niveles se ubicaron en el cuadrante I, y FD, que solo resultó ser activadora tanto en el plano prospectivo como en el retrospectivo en los infantiles. La ubicación de DF y FF no coincidió para ambos niveles de juego. Por un lado, DF resultó ser inhibidora en los planos prospectivo y retrospectivo en infantiles (cuadrante III) y solo en el prospectivo en los cadetes (cuadrante II). Finalmente, la conducta condicionada FF fue inhibitoria en ambos planos para la categoría cadete, mientras que en los infantiles lo fue solo en el retrospectivo. 
Figura 1. Mapa socioconductual para la categoría focal DD (Dentro-Dentro) en relación a las otras categorías del criterio espacio de juego efectivo: DF (Dentro-Fuera), FD (Fuera-Dentro) y FF (Fuera-Fuera).
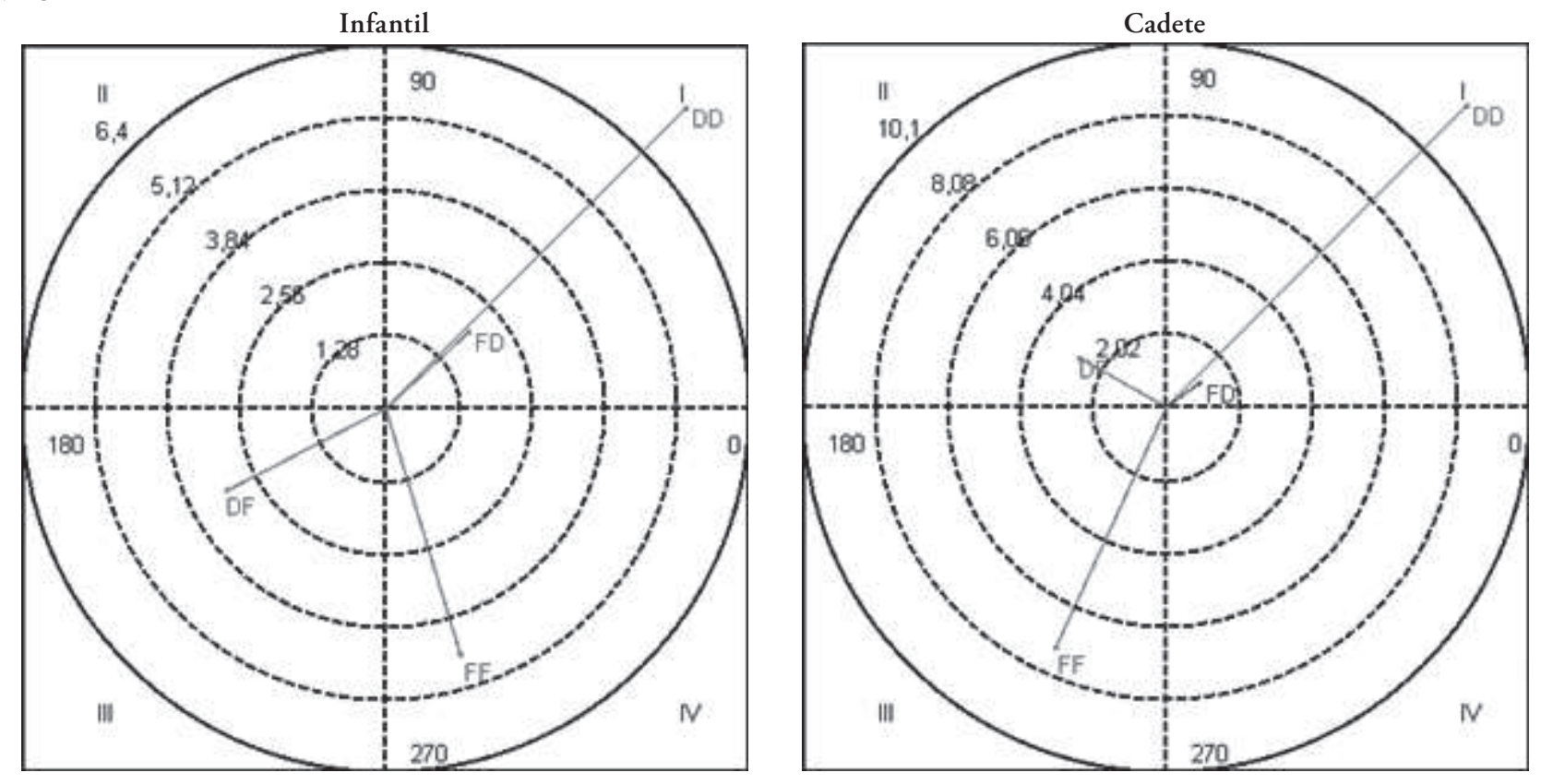

\begin{tabular}{cccccccc}
\hline Nivel & Categoría & Cuadrante & Prosp. & Retrosp. & Ratio & Radio & Ángulo \\
\hline \multirow{6}{*}{ Infantil } & DD & I & 5.3 & 5.3 & 0.71 & 7.5 & 45 \\
& DF & III & -2.84 & -1.47 & -0.46 & 3.2 & 207.33 \\
& FD & I & 1.51 & 1.39 & 0.68 & 2.05 & 42.68 \\
& FF & IV & 1.33 & -4.37 & -0.96 & 4.57 & 286.86 \\
\hline \multirow{6}{*}{ Cadete } & DD & I & 8.44 & 8.44 & 0.71 & 11.93 & 45 \\
& DF & II & -2.52 & 1.42 & 0.49 & 2.89 & 150.53 \\
& FD & I & 1.02 & 0.74 & 0.59 & 1.26 & 35.98 \\
& FF & III & -3.12 & -6.77 & -0.91 & 7.45 & 245.28 \\
\hline
\end{tabular}

Cuando la categoría focal fue DF (Dentro-Fuera) el mapa de relaciones varió también entre infantiles y cadetes (Figura 2), a excepción de DF, situada en ambos casos en el cuadrante I, aunque no de manera significativa. La categoría FF resultó ser activadora en infantiles en los planos prospectivo y retrospectivo (cuadrante I), mientras que en cadetes fue activadora solo en el prospectivo (y con menor intensidad que en los infantiles), e inhibidora en el retrospectivo (cuadrante IV). La categoría DD arrojó ser inhibidora en ambos planos en la edad infantil y activadora en el prospectivo e inhibidora en el retrospectivo para la cadete. Finalmente, la categoría FD llevó dinámicas contrarias según edades, no estableciéndose en ninguna de ellas relaciones significativas. 
Figura 2. Mapa socioconductual para la categoría focal DF (Dentro-Fuera) en relación a las otras categorías del criterio espacio de juego efectivo: DF (Dentro-Fuera), FD (Fuera-Dentro) y FF (Fuera-Fuera).
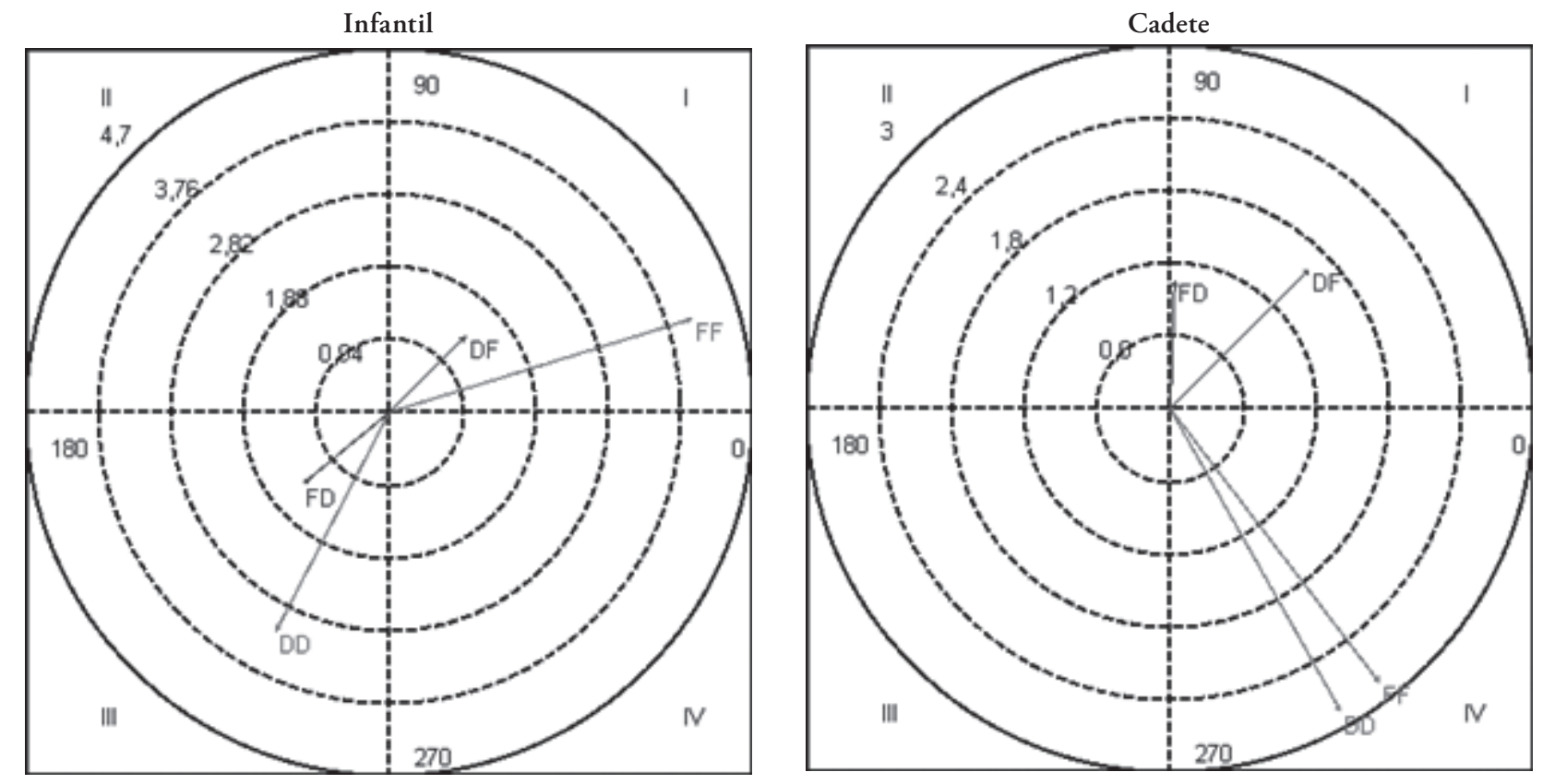

\begin{tabular}{cccccccc}
\hline Nivel & Categoría & Cuadrante & Prosp. & Retrosp. & Ratio & Radio & Ángulo \\
\hline \multirow{4}{*}{ Infantil } & DD & III & -1.47 & -2.84 & -0.89 & 3.2 & 242.67 \\
& DF & I & 0.99 & 0.99 & 0.71 & 1.4 & 45 \\
& FD & III & -1.12 & -0.93 & -0.64 & 1.45 & 219.62 \\
& FF & I & 3.92 & 1.21 & 0.29 & 4.1 & 17.11 \\
\hline \multirow{5}{*}{ Cadete } & DD & IV & 1.42 & -2.52 & -0.87 & 2.89 & 299.47 \\
& DF & I & 1.16 & 1.16 & 0.71 & 1.64 & 45 \\
& FD & I & 0.04 & 1.07 & 1 & 1.07 & 87.92 \\
& FF & IV & 1.74 & -2.28 & -0.8 & 2.86 & 307.33 \\
\hline
\end{tabular}

Los mapas conductuales establecidos a partir de la conducta focal FD (Fuera-Dentro), se presentan en la figura 3. En este caso, ninguna de las relaciones entre conducta focal y las condicionadas resultaron ser significativas en la edad cadete, mientras que en la infantil, FD y DD presentaron relaciones de carácter significativo en ambas perspectivas (cuadrante I). 
Figura 3. Mapa socioconductual para la categoría focal FD (Fuera-Dentro) en relación a las otras categorías del criterio espacio de juego efectivo: DF (Dentro-Fuera), FD (Fuera-Dentro) y FF (Fuera-Fuera).
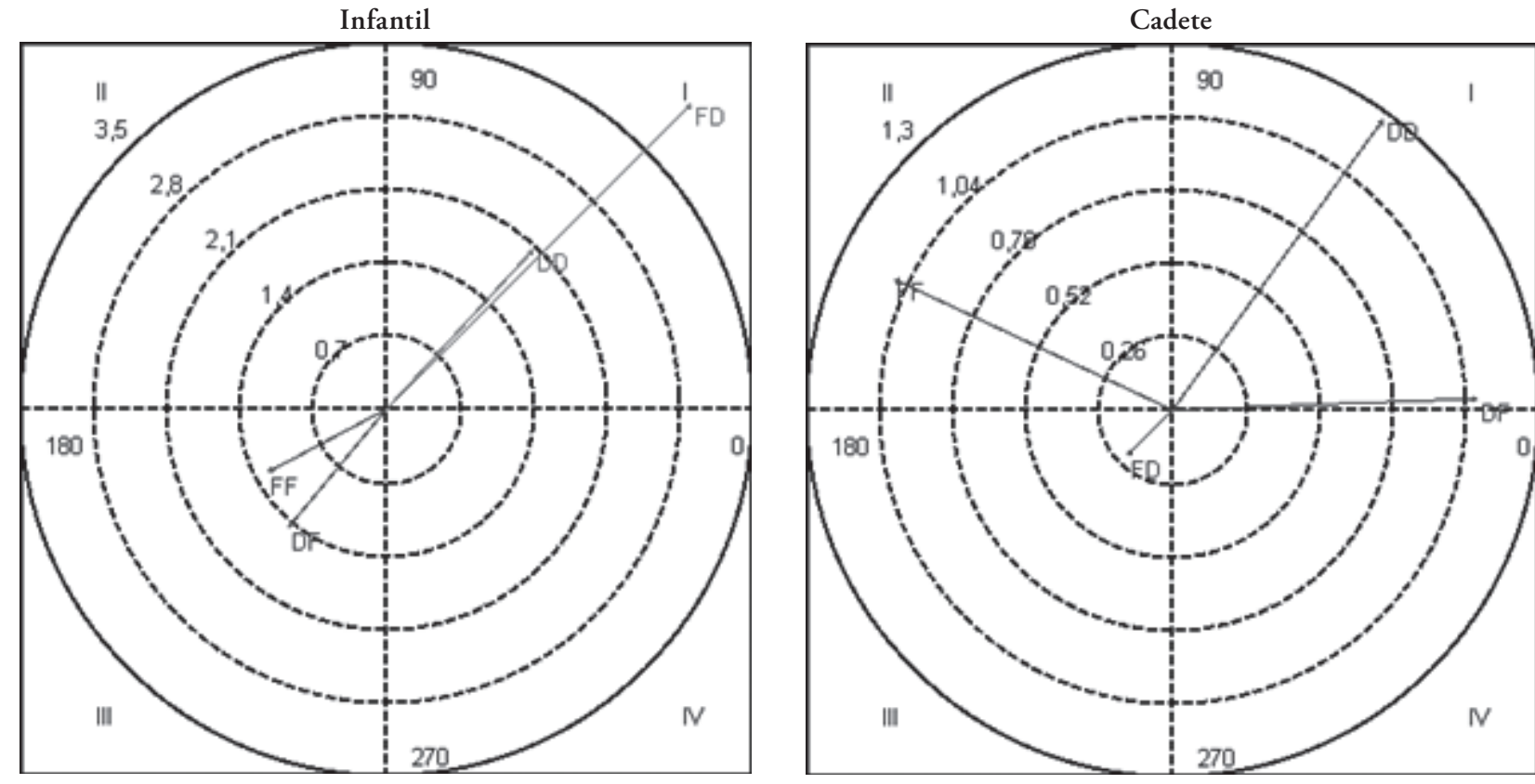

\begin{tabular}{cccccccc}
\hline Nivel & Categoría & Cuadrante & Prosp. & Retrosp. & Ratio & Radio & Ángulo \\
\hline \multirow{6}{*}{ Infantil } & DD & I & 1.39 & 1.51 & 0.74 & 2.05 & 47.32 \\
& DF & III & -0.93 & -1.12 & -0.77 & 1.45 & 230.38 \\
& FD & I & 2.89 & 2.89 & 0.71 & 4.08 & 45 \\
& FF & III & -1.13 & -0.6 & -0.47 & 1.28 & 207.81 \\
\hline \multirow{5}{*}{ Cadete } & DD & I & 0.74 & 1.02 & 0.81 & 1.26 & 54.02 \\
& DF & I & 1.07 & 0.04 & 0.04 & 1.07 & 2.08 \\
& FD & III & -0.16 & -0.16 & -0.71 & 0.23 & 225 \\
& FF & II & -0.98 & 0.46 & 0.42 & 1.08 & 155.1 \\
\hline
\end{tabular}

Finalmente, en la figura 4 se puede observar cómo la categoría FF (Fuera-Fuera) activó la misma conducta criterio o focal (FF) en ambos planos (cuadrante I) aunque con intensidades diferentes para ambos grupos de edad. La categoría FD no se relacionó de manera significativa en ninguna de las edades, mientras que las categorías DF y DD tuvieron una disposición e intensidad diferentes para ambas edades. Por un lado, la categoría DF fue doblemente activadora (cuadrante I) en la categoría de infantiles y solo activadora en el plano retrospectivo (cuadrante II) para los cadetes, mientras que la categoría DD fue inhibitoria en ambos planos en la categoría cadete (cuadrante III) y solo en el plano prospectivo para los infantiles (cuadrante II). 
Figura 4. Mapa socioconductual para la categoría focal FF (Fuera-Fuera) en relación a las otras categorías del criterio espacio de juego efectivo: DF (Dentro-Fuera), FD (Fuera-Dentro) y FF (Fuera-Fuera).

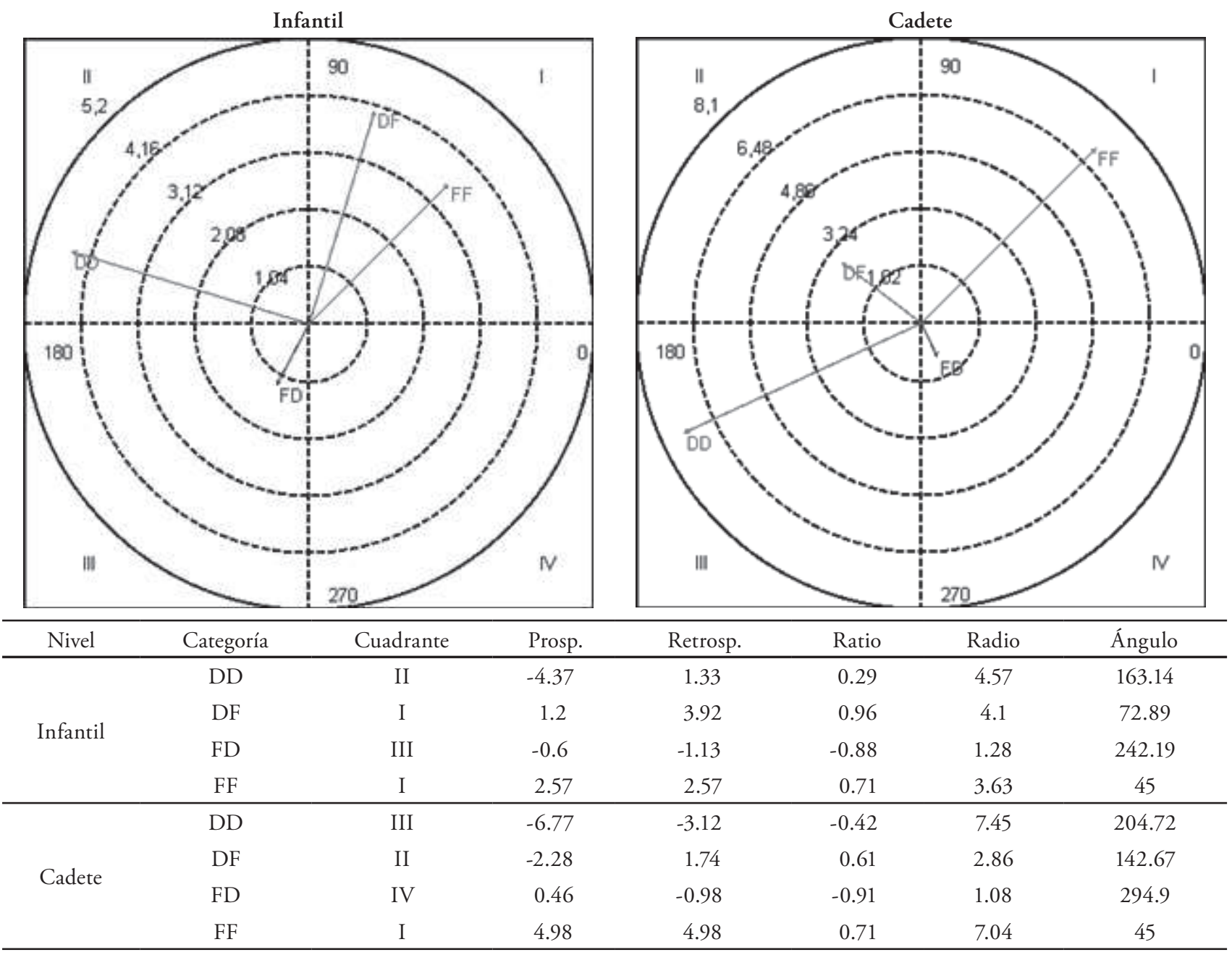

\section{Discusión}

El presente trabajo se ha centrado en conocer el modo en que los jugadores en proceso formativo, categorías infantil y cadete, gestionan el uso espacial como respuesta a la interacción establecida entre jugadores, compańeros y adversarios, en un deporte sociomotor de colaboración-oposición como es el fútbol. Según los datos de los que disponen los autores, ningún trabajo ha aplicado anteriormente en la etapa formativa del fútbol el estudio mediante la aplicación de la técnica de coordenadas polares al concepto de espacio de juego efectivo. Esto ha permitido ahondar en aspectos del juego relativos al espacio de interacción, aportando información acerca del modo en que los jóvenes jugadores de diferentes etapas formativas se disponen en el campo y desarrollan el juego. El principal hallazgo del estudio es que existen diferencias en el tipo de comportamiento colectivo desplegado por los jugadores de categoría infantil y cadete, en relación a las dinámicas en el uso del espacio social.
En primer lugar, se puede observar cómo todas las categorías focales se activan intensamente al relacionarse con ellas mismas (cuadrante I), circunstancia que queda reflejado en ambas categorías de edad. Además, la intensidad de esta relación es mayor en la categoría cadete, lo que podría interpretarse como que los jugadores de mayor edad tienen una mayor competencia en mantener la posesión del balón en comparación con los infantiles. Esto coincide con lo señalado por Almeida, Ferreria y Volossovitch (2013), que sostienen que los jugadores con más experiencia desarrollan un juego ofensivo con secuencias de ataque más largas y con mayor circulación del balón. Siguiendo con la idea de estos mismos autores, la mejor estructura organizativa propia de los jugadores de mayor competencia futbolística, es un factor de especial relevancia que contribuye a este aumento del juego colectivo a medida que se avanza en la edad.

La relación de las propias variables focales consigo mismas es especialmente manifiesta para DD y FF, lo que supone que 
en ambas categorías el mayor número de acciones encadenadas en las posesiones colectivas se realizan desde fuera-fuera y por dentro-dentro, de manera consecutiva. En este sentido, es en estas mismas categorías donde se detecta la mayor diferencia entre grupos de edad, siendo en ambos casos mayor la intensidad de la relaciones DD-DD y FF-FF en cadetes. Esto parece señalar que los cadetes hacen circular más el balón por el perímetro exterior y son capaces de completar posesiones colectivas más largas también por dentro. Con relación a las acciones por fuera, la mayor consistencia en los movimientos colectivos de agrupamiento característica de los jugadores de mayor edad (Folgado, Lemmink, Frencken y Sampaio, 2014), podría dificultar conquistar las zonas interiores, quedando por tanto más protegidas y obligando con ello a circular el balón por fuera para tratar de generar desequilibrios. Con respecto a las acciones por dentro, situaciones que requieren mayor exigencia por el menor espacio y la acumulación de contrarios, la mayor continuidad en el juego en los cadetes podría explicarse, al menos en parte, por la mayor competencia de los jugadores más expertos (González, 2010; Salinero et al., 2013). Sin embargo, la mejora de las estructuras de relación colectivas, también más eficientes en los jugadores más maduros (Sampaio y Maças, 2012), podrían contribuir a explicar las que favorecen el mantenimiento o conservación del balón en el mayor nivel de competencia futbolística de los cadetes podría explicar el hecho de que sean capaces de dar mayor continuidad al jugar por dentro.

Con respecto a los mapas conductuales generados a partir de las conductas focales F-D y D-F, se observan mayores diferencias en la comparativa entre infantiles y cadetes con respecto al tipo e intensidad de las relaciones establecidas. Por un lado, el mayor número e intensidad de las relaciones para los infantiles sugieren una mayor variabilidad en el desarrollo del juego de ataque de los jugadores de esta edad. Esto parece señalar que los infantiles intercalan y alternan en mayor proporción las diferentes alternativas que ofrecen las categorías del criterio EJE, a diferencia de los cadetes, que utilizan una dinámica de juego con menor variabilidad en su desarrollo, donde el peso recae en mayor medida en las situaciones de F-F y D-D. Esta circunstancia podría deberse a la mayor dispersión en el campo, sobre todo en longitud, que caracteriza a los jugadores más jóvenes (Folgado et al., 2014) y que conforme se avanza en la edad, se va equilibrando. De esta manera, el posicionamiento más compacto de los equipos cadetes, dejarían menos alternativas para encadenar situaciones intermedias como DF o FD, prevaleciendo las exclusivamente exteriores e interiores.

En conclusión, en este primer estudio apriorístico, el análisis comparativo del uso del espacio a partir del concepto EJE, e implementando la técnica analítica de coordenadas polares, ha permitido comprobar la existencia de diferencias entre las categorías infantil y cadete, caracterizándose la segunda por una gestión probablemente más eficiente del espacio social. En este sentido, los cadetes muestran una mayor capacidad para circular el balón, especialmente significativa en lo que se refiere a los pases consecutivos realizados por dentro, reflejando una mayor competencia para realizar posesiones más largas. Se observa también en la dinámica de juego de los cadetes un mayor peso y equilibrio en las acciones desarrolladas por fuera y dentro del EJE. Estas diferencias encontradas en el uso del espacio son el resultado de un cambio en la ubicación, distancias y disposición entre jugadores durante el juego, pudiéndose convertir en un indicador de la evolución del nivel de competencia del joven futbolistas, especialmente en un deporte como el fútbol, condicionado tanto por el posicionamiento y movimiento de los jugadores entre sí (Sampaio y Maças, 2012), como por el lugar físico que ocupan en el campo.

\section{Referencias}

1. Almeida, C. H., Ferreira, A. P. y Volossovitch, A. (2013). Offensive Sequences in Youth Soccer: Effects of Experiene and Samll-Sided Games. Journal of Human Kinetics, 36(1), 97-106.

2. Anguera, M. T. (1992). Metodología de la observación en las Ciencias Humanas. Madrid: Cátedra.

3. Anguera, M.T. (1997). From prospective patterns in behavior to joint analysis with a retrospective perspective. Colloque sur invitation "Méthodologied'analyse des interactions sociales». Université de la Sorbonne. Paris.

4. Anguera, M. T. y Losada J. L. (1999). Reducción de datos en marcos de conducta mediante la técnica de coordenadas polares. En M. T. Anguera (Ed.), Observación de la conducta interactiva en situaciones naturales: Aplicaciones. Barcelona: E.U.B.

5. Anguera, M. T., Blanco-Villaseñor, A., Hernández-Mendo, A. y Losada, J. (2011). Diseños observacionales: ajuste y aplicación en psicología del deporte. Cuadernos de Psicología del Deporte, 11(2), 63-76.

6. Ardá, A. (1998). Análisis de los patrones de juego en fútbol a siete. Estudio de las acciones ofensivas. Tesis Doctoral. Universidad de la Coruña.
7. Bakeman, R. y Quera, V. (1996). Análisis de la interacción. Análisis secuencial con SDIS y GSEQ. Madrid: RA-MA [http://www.ub.es/ comporta/sg.htm].

8. Bakeman, R., y Quera, V. (2011). Sequential Analysis and Observational Methods for the Behavioral Sciences. Cambridge, UK: Cambridge University

9. Bangsbo, J., Mohr, M. y Krustrup, P. (2006). Physical and metabolic demands of training and match-play in the elite football player. Journal of Sports Sciences, 24(7), 665-674.

10. Barreira, D., Garganta, J., Castellano, J., Prudente, J. y Anguera, M. T. (2014). Evolución del ataque en el fútbol de élite entre 1982 y 2010: aplicación del análisis secuencial de retardos. Revista de Psicología del Deporte, 23(1), 139-146.

11. Blanco-Villaseñor, A., Castellano, J. y Hernández-Mendo, A. (2000). Generalizabilidad de las observaciones de la acción del juego en el fútbol. Psicothema, 12(2), 81-86.

12. Blanco-Villaseñor, A., Castellano, J., Hernández-Mendo, A., SánchezLópez, C. R. y Usabiaga, O. (2014). Aplicación de la TG en el deporte 
para el estudio de la fiabilidad, validez y estimación de la muestra. $R e-$ vista de Psicología del Deporte, 23(1), 131-137.

13. Camerino, O., Chaverri, J., Anguera, M. T. y Jonsson, G. (2012). Dynamics of the game in soccer: Detection of T-patterns. European Journal of Sport Science, 12(3), 216-224

14. Carling, C. (2013). Interpreting physical performance in professional soccer match-play: should we be more pragmatic in our approach? Sports Medicine, 43(8), 655-663.

15. Carling, C., Williams, A. M. y Reilly, T. (2005). Handbook of soccer match analysis: a systematic approach to improving performance. London: Routledge.

16. Casamichana, D., Castellano, J., Blanco-Villaseñor, A. y Usabiaga, O. (2012). Estudio de la percepción subjetiva del esfuerzo en tareas de entrenamiento en fútbol a través de la teoría de la TG. Revista de Psicologia del Deporte, 21(1), 35-40.

17. Castellano, J. (2000). Observación y análisis de la acción de juego en fútbol. Tesis Doctoral. Universidad del País Vasco.

18. Castellano, J. y Hernández-Mendo, A. (2002). Aportaciones del análisis de coordenadas polares en la descripción de las transformaciones de los contextos de interacción defensivos. Kronos, 1, 42-48.

19. Castellano, J. y Hernández-Mendo, A. (2003). El análisis de coordenadas polares para la estimación de relaciones en la interacción motriz en fútbol. Psicothema, 15(4), 569-574.

20. Castellano, J., Hernández-Mendo, A. y Haro, J. A. (2002). Mapas socioconductuales de la selección francesa en el Mundial de Francia'98. Revista de Psicología del Deporte. 11(1), 35-51.

21. Castellano, J., Hernández-Mendo, A., Morales-Sánchez, V. y Anguera, M. T. (2007). Optimising a probabilistic model of the development of play in soccer. Quality \& Quantity, 41(1), 93-104.

22. Castellano, J., Perea, A., Alday, L. y Hernández-Mendo, A. (2008). Measuring and Observation Tool in Sports. Behavior Research Methods, 40(3), 898-903.

23. Castellano, J., Perea, A. y Blanco-Villaseñor, A. (2007). Has soccer changed in the last three world championship?. Journal of Sports science \& Medicine, 6(10), 2.

24. Castellano, J., Perea, A. y Hernández-Mendo, A. (2007). Diachronic analysis of interaction context in '06 World Championship. Journal of Sports Science \& Medicine, 6(10), 200-201.

25. Castellano, J., Perea, A. y Hernández-Mendo, A. (2008). Análisis de la evolución del fútbol a lo largo de los mundiales. Psicothema, 20(4), 928-932.

26. Davids, K., Araújo, D. y Shuttleworth, R. (2005). Applications of dynamical systems theory to football. In T. Reilly, J. Cabri y D. Araújo (Eds.), Science and football V, (pp.537-550). London: Routledge, Taylor \& Francis.

27. Duarte, R., Araújo, D., Fernandes, O., Fonseca, C., Correia, V., Gazimba, V., Travassos, B., Esteves, P. y José Lopes, L. V. (2010). Capturing complex human behaviors in representative sports contexts with a single camera. Medicina (Kaunas), 46(6), 408-414.

28. Echeazarra, I. (2014). Análisis de la respuesta fisica y del comportamiento motor en competición, en futbolistas de categoría alevin, infantil y cadete. Tesis Doctoral. Vitoria: Universidad del País Vasco.

29. Echeazarra, I., Castellano, J. y Usabiaga, O. (2013). Aplicación de diferentes estrategias para el control de calidad del dato de una herramienta observacional en fútbol formación. Revista Iberoamericana de Psicología del Ejercicio y el Ddeporte, 8(2), 65-80.

30. Folgado, H., Lemmink, K., Frencken, W. y Sampaio, J. (2014). Length, width and centroid distance as measures of teams tactical performance in youth football. European Journal of Sport Science, 14(1), 487-492.

31. González, S. (2010). Estudio de las etapas de formación del joven deportista desde el desarrollo de la capacidad táctica. Aplicación al fútbol. Tesis Doctoral. Cuenca: Universidad de Castilla-La Mancha.

32. Grèhaigne, J-F. (1992). L'organisation du jeu en football. Paris: Actio.
33. Grèhaigne, J-F., Bouthier, D. y David, B. (1997). Dynamic-system analysis of opponent relationships in collective actions in soccer. Journal of Sport Sciences, 15(2), 137-149.

34. Gréhaigne, J-F., Godbout, P. y Zerai, Z. (2011). How the "rapport de forces" evolves in a soccer match: the dynamics of collective decisions in a complex system. Revista de Psicología del Deporte, 20(2),747-765.

35. Hernández-Mendo, A. (1996). Observación y análisis de patrones de juego en deportes sociomotores. Tesis Doctoral. Santiago de Compostela: Universidad de Santiago de Compostela.

36. Hernández-Mendo, A. y Anguera, M.T. (1998). Análisis de coordenadas polares en el estudio de las diferencias individuales de la acción de juego. En: Sánchez, M.P., López-Quiroga Estévez, M.A. (eds.). Perspectivas actuales en la investigación de las diferencias individuales, (pp. 84-88). Madrid: Centro de Estudios Ramón Areces.

37. Hernández-Mendo, A. y Anguera, M. T. (1999). Aportaciones de análisis de coordenadas polares a los deportes de equipo. En Félix Guillén García, La Psicología del Deporte en España al final del Milenio (pp.169175). Las Palmas de Gran Canaria: Servicio de Publicaciones y Producción Documental de la Universidad de Las Palmas de Gran Canaria.

38. Hernández-Mendo, A., López-López, J. A., Castellano, J., MoralesSánchez, V. y Pastrana, J. L. (2012). HOISAN 1.2: Programa informático para uso en metodología observacional. Cuadernos de Psicología del Deporte, 12(1), 55-78.

39. Hernández-Mendo, A., Ramos-Pérez, F. y Pastrana, J. L. (2012). SAGT: Programa informático para análisis de Teoría de la Generalizabilidad. SAFE CREATIVE Código: 1204191501059.

40. Horn, R. (2002). World-wide review of Science and football research. Insight, 4(4), 20-22.

41. Morris, T. (2000). Psychological characteristics and talent identification in soccer. Journal of Sport Sciences, 18(9), 715-726.

42. Perea, A. (2008). Análisis de las acciones colectivas en el fútbol de rendimiento. Tesis Doctoral. Vitoria: Universidad del País Vasco.

43. Perea, A., Alday, L. y Castellano, J. (2007). Programación del análisis de la técnica de coordenadas polares a partir del matlab. En J. Castellano y O. Usabiaga (Eds.), Investigación en la Actividad Física y el Deporte II (pp. 193-204). Vitoria-Gasteiz: Facultad de Ciencias de la Actividad Física y el Deporte.

44. Perea, A., Castellano, J., Alday, L. y Hernández-Mendo, A. (2012). Analysis of behaviour in sports through Polar Coordinate Analysis with MATLAB'. Quality \& Quantity, 46(4), 1249-1260.

45. Reilly, T. (2009). Science and football. An update. En T. Reilly y F. Korkusuz (Eds.), Science and Football VI (pp. 3-8). London: Routledge.

46. Robles, J. F. (2012). Observación y análisis de las acciones ofensivas de la selección española de fútbol en la Eurocopa de 2008 y en el Mundial de 2010. Tesis doctoral. Vitoria: Universidad del País Vasco.

47. Robles, J. F. y Castellano, J. (2012). Comparación entre el juego ofensivo de la selección española de fútbol y sus rivales en la Eurocopa'08 y Mundial'10. Revista Iberoamericana de Psicología del Ejercicio y el Deporte, 7(2), 307-322.

48. Robles, F. J. y Castellano, J. (2014). Comparación entre el juego ofensivo de la selección española de fútbol y sus rivales en la Eurocopa '08 y Mundial '10. Revista Iberoamericana de Psicología del Ejercicio y el Deporte, 7(2), 323-338.

49. Robles, F., Castellano, J., Perea, A., Martínez-Santos, R. y Casamichana, D. (2011, May). Spatial strategy used by the world champion in south Africa'10. 7th World Congress on Science \& football, May 26-30, Nagoya (Japan).

50. Sackett, G. P. (1980). Lag Sequential Analysis as a data Reduction Technique in Social Interaction Research. En D. B. Sawin, R.C. Hawkins, L. O. Walker y J. H. Penticuff (eds.), Exceptional infant. Phychosocial risks in infant-environment transactions (pp. 300-340). New York: Brunner/Mazel.

51. Salinero, J. J., González, C., Ruiz, D., Vicen, J. A., García, A., Rodrí- 
guez, M. y Cruz, A. (2013). Physical fitness and technique evaluation in young soccer players. Revista Internacional de Medicina y Ciencias de la Actividad Fisica y el Deporte, (13)50, 401-418.

52. Sampaio, J. y Maçãs, V. (2012). Measuring tactical behaviour in football. International Journal of Sports Medicine, 33(5), 395-401.

53. Seabra, F. y Dantas, L. E. P. B. T. (2006). Space definition for match analysis in soccer. International Journal of Performance Analysis in Sports, 6(2), 97-102.

54. Suzuki, K. y Nishijima, T. (2004). Validity of a soccer defending skill scale (SDSS) using game performance. International Journal of Sport and Health Science, 2, 34-49.
55. Usabiaga. O., Castellano, J., Blanco-Villaseñor, A. y Casamichana, D. (2013). La Teoría de la Generalizabilidad en las primeras fases del método observacional aplicado en el ámbito de la iniciación deportiva: calidad del dato y estimación de la muestra. Revista de Psicología del Deporte, 22(1), 103-109.

56. Vegas, G. (2006). Metodología de enseñanza basada en la implicación cognitiva del jugador de fútbol base. Tesis Doctoral. Granada: Universidad de Granada.

57. Wein, H. (2007). Developing Youth Soccer Players. Champaign IL: Human Kinetics. 